\title{
Neuromodulatory systems
}

\author{
Gerhard Werner ${ }^{1 \dagger}$ and Bernhard J. Mitterauer ${ }^{2 *}$ \\ ${ }^{1}$ Department of Biomedical Engineering, University of Texas, Austin, TX, USA \\ ${ }^{2}$ Forensic Psychiatry and Guenther Archives, University of Salzburg, Wals, Austria
}

\section{Edited by:}

Paul S. Katz, Georgia State

University, USA

Reviewed by:

Paul S. Katz, Georgia State

University, USA

Vladimir Brezina, Mount Sinai School

of Medicine, USA

\section{*Correspondence:}

Bernhard J. Mitterauer, Gotthard

Guenther Archives,

Volitronics-Institute for Basic

Research, Psychopathology and

Brain Philosophy, University of

Salzburg, Autobahnweg 7,

Wals A-5071, Austria.

e-mail: mitterauer@wasi.tv

${ }^{\dagger}$ Gerhard Werner died before the

final version of this manuscript was

submitted.
We examine the interactions and interdependencies between Neuroglia, the Brain-Cell Microenvironment, and the processes commonly subsumed under Neuromodulation. The interactions of the component processes covering a wide spectrum of frequencies are designated as Neuromodulatory Systems (NMS). This implies NMS's scale-invariance as the capacity of linking actions across many time scales, and self-similarity at any scale. These features endow NMS with the ability to respond adaptively to neural impulse traffic of an unpredictably wide frequency spectrum. In this preliminary perspective, the components of NMS are only outlined based on concepts of Complex Systems Dynamics. However, their interactions must be formally elaborated in further investigations.

Keywords: neuroglia, extracellular fluid, neuromodulation, multifractals, neuromodulatory systems

\section{INTRODUCTION}

We address three aspects of neuroscience, each for long being largely overshadowed by the Neuron Doctrine's hegemony (Bullock et al., 2005): Neuroglia (including here also the systems of neuronal and glial Gap Junctions), Extracellular Fluid in neural tissue ("Brain-Cell Microenvironment": Nicholson, 1980), and neuromodulatory processes. Our leading notion is that the functional state of neurons, individually and in assemblies, is determined by a set of variables (ion conductances and membrane currents, thresholds for neural discharges, synaptic potentials, ion channel kinetics, etc.), whose values at any one time are to varying degrees affected by interactions and interdependencies of these three components, locally as well as globally, and at largely different time scales. In the section "Background," we review essential aspects of each of these components separately. This is to provide the basis for our principal objective to analyze in the "Discussion" section the global dynamics of the complex system these components jointly compose, covering a wide range of temporal scales that is characteristic of Multifractals. Accordingly, self-similarity and the absence of any specific time scale ensure instant and automatic adaptation to neural impulse traffic over a wide range of frequencies.

\section{BACKGROUND}

\section{GAP JUNCTIONS AND NEUROGLIA}

Diffusive coupling by Gap Junctions between various interneuron types and neuroglia from cells in neocortex is now firmly established (Simon et al., 2005), as is their virtually boundless distribution (Fukuda, 2007). Simulation studies determined their role for supporting synchronous oscillations (Traub et al., 1999; Lewis and Rinzel, 2000), and identified complementary interrelations with chemical synapses in interneuronal networks (Kopell and Ermentrout, 2004). Electrical coupling between axons is also amply documented (Debanne and Rama, 2011), providing the opportunity for fast and efficient transfer of action potentials for generating highly coherent output pathways of neuronal networks.

Neurochemists generated an avalanche of data, promoting Astrocytes (Verkhratsky and Butt, 2007), one of the members of the macro-glia family, to full partnership with pre- and postsynaptic neurons in the "Tripartite Synapse" (Araque et al., 1999). This has become a fertile concept for characterizing the complex and reciprocal patterns of interactions between astrocytes and neurons, reviewed by Araque and Navarrete (2010) and Halassa and Haydon (2010). The dynamics of these interactions is sustained by the astrocytes expressing receptors for virtually all important neurotransmitters (Kettenmann and Steinhauser, 2005), providing multiple opportunities for engaging neuronastrocyte complexes at many target points (Fields and StevensGraham, 2002; Auld and Robitaille, 2003; Perea et al., 2009). A prevalent feature of astrocytes' role is their participation in the dynamics of calcium in extracellular space on two different space and time scales. For local short-term interaction, astrocytes (although not electrically excitable) respond to glutamate liberated at presynaptic junctions with calcium spikes which, in turn, release additional glutamate and ATP to neighboring neurons (Smith and Pereda, 2003) for integrating coincident activity from different dendrites in the same tissue volume (Parpura and Haydon, 2000; Bezzi et al., 2001). Furthermore, activity related changes of calcium levels within astrocytes contribute to mobilizing various transmitters and transmission-related substances (Perea and Araque, 2010). Globally and on longer time scales, intercellular propagation of Calcium waves (Cornell-Bell et al., 1990; Charles, 1998; Harris and Timofeeva, 2010) can support 
long-range signaling (Giaume and Venance, 1998; Kuga et al., 2011). Recent evidence from the family of connexins suggest that the astrocyte system constitutes a network of communicating cells with definite spatial organization (Pereira and Furlan, 2010) where intercellular communication is controlled by endogenous signals (Giaume and Liu, 2011).

The dynamics of neuron glia interaction is complicated by two circumstances: one, due to activity-dependent morphological changes of astroglia processes ending at synaptic regions (Hirrlinger et al., 2004; Theodosis et al., 2008; Fellin, 2009); and, the second, due to a complex anatomical organization of spatial non-overlapping domains with limited interdigitation of processes from adjacent cells (Bushong et al., 2002; Ogata and Kosaka, 2002; Halassa et al., 2007). Each domain encompasses some 2 million synapses in human brain (Oberheim et al., 2008) as an area of the neuropil that is controlled by a single astrocyte. Moreover, parts of this territory can be controlled autonomously by specialized astrocyte microdomains of filipodia with distinct motility (Volterra and Meldolesi, 2005). Groups of neurons are also enwrapped by a layer of lattice-like material: this Perineuronal Net forms stable complexes surrounding synapses (Faissner et al., 2010; Kwok et al., 2011), seemingly affecting short-term synaptic plasticity (Frischknecht et al., 2009). On a modular basis, computational simulations of different manifestations of astrocyte-neuron interactions contribute to gaining some insight into their functions (Nadkarni et al., 2008; Goldberg et al., 2010; De Pitta et al., 2011). On the basis of theoretical arguments, Mitterauer (2012) attributed a structural organization in the form of logical functions to the tripartite synapse and astrocyte domain organization, suggesting its role in the economy of normal and pathological brain functions (Mitterauer, 2010; Mitterauer and Kofler-Westergren, 2011).

\section{BRAIN-CELL MICROENVIRONMENT (EXTRACELLULAR FLUID)}

Extracellular fluid's coming-of-age is closely associated with the work of Kuffler and Nicholls (1966) that identified the diffusion of ions and various neuroactive substances in intercellular clefts of neural tissue. This theme was again taken up by Vizi and Labos (1991), documenting non-synaptic interaction in nervous tissue, subsequently discussed in detail by Agnati et al. (1995) and Zoli and Agnati (1996), suggesting to view intercellular communication among cells in the nervous system in two complementary reference frames, one as "wiring" transmission, the other as "volume" transmission: the former being transmission of excitation between synaptically connected neurons, the latter attributing diffusive distribution of various ions, neuropeptides, and neurotransmitters to extracellular fluid surrounding neurons. A wealth of experimental data, notably with the effects of ion accumulation in the extracellular fluid following tetanic nerve activity corroborated this conjecture (Frankenhaeuser and Hodgkin, 1956; Egelman and Montague, 1998). In a computational model of a Reaction-Diffusion system, Werner (2005) demonstrated that tetanic stimulation of a group of neurons causes extracellular accumulation of Calcium ions which induces spreading activity patterns in surrounding unstimulated neurons. More recently, Froehlich et al. (2006) showed that diffusive modulation of extracellular potassium concentration induces state transitions in neurons with distinct changes in oscillatory patterns. Changes of diffusive coupling in neural networks can change normal, and precipitate pathological, activity patterns (Ullah et al., 2009; Durand et al., 2010). The relevance of nonsynaptic diffusion neurotransmission was further extended and refined by Bach-Y-Rita (1995). However, diffusion of neuroactive substances is slowed down by geometric tortuosity and viscosity of macromolecules in extracellular space (Rusakov and Kullmann, 1998; Hrabe et al., 2004). By changing their geometric shape, dendritic spines can dynamically regulate diffusion in their vicinity (Biess et al., 2007).

\section{NEUROMODULATORY PROCESSES}

The notion of Neuromodulation originated with a diversity of observations that could not be accounted for within the established principles of synaptic transmission with transmitter substances exclusively acting locally at synaptic sites (Kaczmarek and Levitan, 1987; Katz, 1999). For present purposes, we suggest reserving the term "Neuromodulation" to designate the composite system of all processes affecting synaptic transmission, in distinction from individual neuromodulatory processes in the narrower sense, as listed in the following. The foundational insights on operational principles of neuromodulation originated with investigating the polyvalence of neural network functions in crustaceans. A succession of comprehensive reviews by Getting (1989), Harris-Warrick and Marder (1991), and Marder and Calabrese (1996), summarize the repertoire of neuronal functions attributable to the modulating action of extrasynaptic processes on synaptic transmission. They include effects on synaptic efficacy and presynaptic transmitter release, intrinsic neuronal properties, changes of network connectivity, coupling of neural oscillators, and filtering sensory input, and spike-time dependent plasticity (Pawlak et al., 2010). All known neurotransmitter substances are involved in these effects, as are a multitude of peptides (Nusbaum, 2002; Nassel, 2009).

Reports of neuromodulatory effects in higher functions of vertebrates followed the crustacean work in rapid sequence: Hasselmo and associates produced evidence for forebrain cholinergic neuromodulation of Cognition (for review see: Hasselmo and Sarter, 2011). Ascending brain stem neuromodulatory systems (NMS) of vertebrate brains became implicated in learning mechanisms (Doya, 2002), in adaptive behavior (Krichmar, 2008), and in emotional control processes (for a recent example, see Cools et al., 2007). Central pattern generators are subject to neuron modulation in vertebrates as they are in invertebrates (Dickinson, 2006). In its totality, the accumulated observational evidence mandates expanding the classical view of a relatively static neuronal "wiring diagram" to a dynamic system subject to ongoing tuning and reconfiguring by a biochemical network of modulators, effective over a wide range of temporal, and spatial scales (Brezina, 2010). Combining experimental observations with computational simulations reveals the combinatorial richness of the modulatory network for generating functionally appropriate and adaptive behavior (Brezina et al., 2000; Proekt et al., 2004; Stern et al., 2007).

The multitude of phenomena described as neuromodulation fall into two fundamentally different categories (Marder and 
Thirumalai, 2002): intrinsic neuromodulation is the condition of the modulator being released by some of the same neurons that are also part of the circuit they modulate (Katz and Frost, 1996). Hansson and Rönnbäck (1994) review several instances of intrinsic modulation of synaptic transmission by astrocytes, related to release and uptake of glutamine at synaptic sites. Events at the "Tripartite Synapse" fall also in this category. Extrinsic modulation, on the other hand, consists in activity of functionally distinct system processes outside of and parallel to the actual synaptic activity, relying on the storage and transport of neuroactive substances in the extracellular fluid compartment. Most of the effects of neuroglia must be attributed to this category. Beyond regulating merely one synaptic region, extrinsic modulation can globally organize ensembles of circuits, and usually works at a time course up to several seconds rather than the msec's of synaptic actions of intrinsic modulation.

\section{DISCUSSION AND CONCLUSION}

In the following discussion, we will refer to the totality of the interacting complex of glia, extracellular fluid and the processes of neuromodulation as NMS. For formulating ideas about NMS, it must of course not be overlooked that neurons themselves (individually and as assemblies) are integral participants, active by contributing to the flux of neuroactive substances in extracellular space, and passive by being affected by them. Since our aim is to characterize the function of NMS at a global level, we take a coarse-grained, non-reductive perspective. This sets our approach apart from studies of stochastic synaptic processes at the molecular level (Ribrault et al., 2011) and the multiscale analysis of molecular processes at cellular levels (for a recent overview: see Holcman, 2012).

We make the biologically plausible assumption that each process in the chain of neuromodulatory events can be considered a chemical rate process with exponential decay. Relaxation rates vary over at least a thousand-fold range: from milliseconds at the liberation of transmitter substances at intrinsic modulation, to many seconds of chemically mediated astrocyte network reconfigurations and propagating calcium waves, with the numerous extrinsic modulatory processes exhibiting intermediate rates. This situation invites applying the observation of Hausdorff and Peng (1996) that systems presenting time series with widely differing scales of component regulatory mechanisms summate to a system's power-law (1/f) scaling, suggestive of its fractal character. Although there is no definitive mathematical proof presently available that time-scale free functions emerge from superposition of independent relaxation processes, there exists a range of physical mechanisms that do in fact show such micro- to macroscopic conversion, generally in the context of fractal time series (Marom, 2010). Moreover, numerical analyses of Montroll and Shlesinger (1982) established that macroscopic scale-free functions emerge, provided the independent microscopic relaxation processes are of sufficiently large variance, as they are in NMS. This principle was subsequently applied by Anderson (2001) to ascertain the power-law dependency as an emergent property of systems that contain several exponentially decaying traces and was further extended by Fusi et al. (2005) and Drew and Abbott (2006) to include cascading exponential processes, the latter for sensory adaptation. However, NMS contain too many unknown rate constants to attempt numerical simulation and determination of a power law exponent. Thus, we need to confine the discussion to the exposition of plausible principles and analogies.

Placing the function of NMS into the domain of fractal time series allows gaining significant insights into the dynamic properties. In the first place, the scale-invariance is identified as the property of relating the behavioral elements of NMS in time across multiple time scales. This is a characteristic empirical feature of a large number of complex physiological phenomena (West, 2010). It implies the global system's capacity for linking actions across many time different scales of the constituent processes: there is no privileged time scale, and the system's temporal performance is self-similar at any scale. This property endows the system with the ability to respond adaptively to perturbations (external events) over a wide range of their temporal patterns, and enables adaptation to impinging neural impulse trains that vary unpredictably over a wide range of time scales (Werner, 2010,2011 ). However, if the special formal properties of selfsimilarity, etc. are not present, then what we are left with is a collection of processes on multiple time scales, which can therefore respond to perturbations on various time scales individually, but not necessarily as a coherent system.

In the application cited in the foregoing, the systems were sufficiently small that power-laws with only one exponent were considered adequate. Hence, they fall into the category of Monofractals. Granting, however, the plausibility of the suggested approach, we consider it necessary to introduce a refinement: characteristic time scales of the NMS component processes known to extend over a thousand fold range (as stated earlier) render fitting a power-law function with only one exponent unlikely. Accordingly, several power-functions with different exponents, each covering a section of the entire spectrum of scales, are required. This places NMS into the category of Multifractals (Stanley and Meakin, 1988; Mandelbrot, 1999), commonly thought indispensable for very large systems (for instance: Geophysics: Mandelbrot, 1999), but also successfully applied in numerous biological systems (West, 2010; West and Grigolini, 2010). This underscores the wide range of temporal scales to which systems with fractal characteristic can successfully adapt (Werner, 2010).

\section{A FINAL THOUGHT}

Given the function NMS are to satisfy the requirements stipulated in the foregoing, it is from an engineering point of view surely extremely clumsily designed, with many redundancies and duplications of functions. Why is this so? It gives the impression that NMS in the present state may represent stages, one stage superimposed on the other as if to attain a progressively higher degree of robustness and stability for assuring secure contact with an ever-changing and unpredictable environment: perhaps many stages of consecutive "tinkering"; yet, seemingly preserving modular semi-autonomy. Admittedly, in this first perspective the components of NMS are only outlined. Their interactions must be formally elaborated in further investigations. 


\section{REFERENCES}

Agnati, L. F., Zoli, M., Stromberg, I., and Fuxe, K. (1995). Intercellular communication in the brain: wiring versus volume transmission. Neuroscience 69, 711-726.

Anderson, R. B. (2001). The power law as an emergent property. Mem. Cogn. 29, 1061-1068.

Araque, A., and Navarrete, M. (2010). Glial cells in neuronal network function. Philos. Trans. R. Soc. Lond. B Biol. Sci. 365, 2375-2381.

Araque, A., Parpura, V., Sanzgiri, R. P., and Haydon, P. G. (1999). Tripartite synapses: glia, the unacknowledged partner. Trends Neurosci. 22, 208-215.

Auld, D. S., and Robitaille, R. (2003). Glial cells and neurotransmission: an inclusive view of synaptic function. Neuron 40, 389-400.

Bach-Y-Rita, P. (1995). Nonsynaptic Diffusion Neurotransmission and Late Brain Reorganization. New York, NY: Demos Medical Publishing.

Bezzi, P., Domercq, M., Vesce, S., and Volterra, A. (2001). Neuronastrocyte crosstalk during synaptic transmission. Prog. Brain Res. 132, 255-265.

Biess, A., Korkotian, E., and Holcman, D. (2007). Diffusion in a dendritic spine: the role of geometry. Phys. Rev. E 76, 021922.

Brezina, V. (2010). Beyond the wiring diagram: signaling through complex neuromodulator networks. Philos. Trans. R. Soc. Lond. B Biol. Sci. 365, 2363-2374.

Brezina, V., Orekhova, I. V., and Weiss, K. R. (2000). Optimization of rhythmic behaviors by modulation of the neuromuscular transform. J. Neurophys. 83, 260-279.

Bullock, T. H., Bennett, M. V. L., Johnston, D., Josephson, R., Marder, E., and Fields, R. D. (2005). The neuron doctrine, redux. Science 310 , 791-793.

Bushong, E. A., Martone, M. E., Jones, Y. Z., and Ellisman, M. H. (2002). Protoplasmic astrocytes in CA1 stratum radiatum occupy separate anatomical domains. J. Neurosci. 22, 183-192.

Charles, A. (1998). Intercellular calcium waves in glia. Glia 24, 39-49.

Cools, R., Roberts, A. C., and Robbins, T. W. (2007). Serotonergic regulation of emotional and behavioural control processes. Trends Cogn. Sci. 12, 31-40.

Cornell-Bell, A. H., Finkbeiner, S. M., Cooper, M. S., and Smith, S. J. (1990). Glutamate induces calcium waves in cultured astrocytes: long-range glial signaling. Science 247, 470-473.

Debanne, D., and Rama, S. (2011). Astrocytes shape axonal signaling. Sci. Signal. 4:162. doi: 10.1126/ scisignal.2001884

De Pitta, M., Volman, V., Berry, H., and Ben-Jacob, E. (2011). A tale of two stories: astrocyte regulation of synaptic depression and facilitation. PLoS Comput. Biol. 7:e1002293. doi: 10.1371/journal.pcbi.1002293

Dickinson, P. S. (2006). Neuromodulation of central pattern generators in invertebrates and vertebrates. Curr. Opin. Neurobiol. 16, 604-614.

Doya, K. (2002). Metalearning and neuromodulation. Neural Netw. 15, 495-506.

Drew, P. J., and Abbott, L. F. (2006). Models and properties of powerlaw adaptation in neural systems. J. Neurophysiol. 96, 826-833.

Durand, E. M., Park, E. H., and Jensen, A. L. (2010). Potassium diffusive coupling in neural networks. Philos. Trans. R. Soc. Lond. B Biol. Sci. 365, 2347-2362.

Egelman, D. M., and Montague, P. R. (1998). Computational properties of peri-dendritic calcium fluctuations. J. Neurosci. 18, 8580-8589.

Faissner, A., Pyka, M., Geissler, M., Sobik, T., Frischknecht, R., Gundelfinger, E. D., et al. (2010). Contributions of astrocytes to synapse formation and maturation - potential functions of the perisynaptic extracellular matrix. Brain Res. Rev. 63, 26-38.

Fellin, T. (2009). Communication between neurons and astrocytes: relevance to the modulation of synaptic and network activity. J. Neurochem. 108, 533-544.

Fields, R. D., and Stevens-Graham, B. (2002). New insights into neuronglia communication. Science 298, 551-562.

Frankenhaeuser, B., and Hodgkin, A. L. (1956). The aftereffects of impulses in the giant nerve fibers of Loligo. J. Physiol. 341-376.

Frischknecht, R., Heine, M., Perrais, D., Seidenbecher, C. I., Choquet, D., and Gundelfinger, E. D. (2009). Brain extracellular matrix affects AMPA receptor lateral mobility and short-term synaptic plasticity. Nat. Neurosci. 12, 897-904

Froehlich, F., Bazhenov, M., Timofeev, I., Steriade, M., and Sejnowski, T. J. (2006). Slow state transitions of sustained neural oscillations by activity-dependent modulation of intrinsic excitability. J. Neurosci. 26, 6153-6162.
Fukuda, T. (2007). Structural organization of the gap junction in the cerebral cortex. Neuroscientist 13 199-207.

Fusi, S., Drew, P. J., and Abbott, L. F. (2005). Cascade models of synaptically stored memories. Neuron 45, 599-611.

Getting, P. A. (1989). Emerging principles governing the operation of neural networks. Annu. Rev. Neurosci. 12, 185-204.

Giaume, C., and Liu, X. (2011). From glial syncytium to a more restricted and specific glial networking. J. Physiol. Paris. 106, 34-39.

Giaume, C., and Venance, L. (1998). Intercellular calcium signaling and gap junctional communication in astrocytes. Glia 24, 50-64.

Goldberg, M., Pitta, M. D., Volman, V., Berry, H., and Ben-Jacob, E. (2010). Nonlinear gap junctions enable long-distance propagation of pulsating calcium waves in astrocyte networks. PLoS Comput. Biol. 6:e1000909. doi: 10.1371/journal. pcbi.1000909

Halassa, M. M., Fellin, T., Takano, H. Dong, J.-H., and Haydon, P. G. (2007). Synaptic islands defined by the territory of a single astrocyte. J. Neurosci. 27, 6473-6477.

Halassa, M. M., and Haydon, P. G. (2010). Integrated brain circuits: astrocytic networks modulate neuronal activity and behavior. Annu. Rev. Physiol. 72, 335-355.

Hansson, E., and Rönnbäck, L. (1994) Astroglial modulation of synaptic transmission. Perspect. Dev. Neurobiol. 2, 217-223.

Harris, J., and Timofeeva, Y. (2010) Intercellular calcium waves in the fire-diffuse-fire framework: Green's function for gap junctional coupling. Phys. Rev. E 82, 051910.

Harris-Warrick, R. M., and Marder, E. (1991). Modulation of neura networks for behavior. Annu. Rev. Neurosci. 14, 39-57.

Hasselmo, M. E., and Sarter, M. (2011). Modes and models of forebrain cholinergic neuromodulation of cognition. Neuropsychopharmacology 36 52-73.

Hausdorff, J. M., and Peng, C.-K. (1996). Multiscaled randomness: a possible source of $1 / \mathrm{f}$ noise in biology. Phys. Rev. E 54, 2154-2157.

Hirrlinger, J., Hulsmann, S., and Kirchhoff, F. (2004). Astroglial processes show spontaneous motility at active synaptic terminals in situ. Eur. J. Neurosci. 20, 2235-2239.

Holcman, D. (2012). The complexity of synaptic transmission revealed by a multiscale analysis approach from the molecular to the cellular level. Available online at: http://arxiv.org/abs/1202.2034

Hrabe, J., Hrabetova, S., and Segeth, K. (2004). A model of effective diffusion and tortuosity in the extracellular space of the brain. Biophys. J. 87, 1606-1617.

Kaczmarek, L. K., and Levitan, J. B. (1987). Neuromodulation. New York, NY: Oxford University Press.

Katz, P. S. (1999). Beyond Neurotransmission: Neuromodulation and its Importance for Information Processing. Oxford: Oxford University Press.

Katz, P. S., and Frost, W. N. (1996). Intrinsic neuromodulation: altering neuronal circuits from within. Trends Neurosci. 19, 54-61.

Kettenmann, K., and Steinhauser, C. (2005). "Receptors for neurotransmitters and hormones," in Neuroglia, eds K. Kettenmann and B. R. Ransom (Oxford: Oxford University Press), 131-145.

Kopell, N., and Ermentrout, B. (2004). Chemical and electrical synapses perform complementary roles in the synchronization of interneuronal networks. Proc. Natl. Acad. Sci. U.S.A. 101, 15482-15487.

Krichmar, J. L. (2008). The neuromodulatory system: a framework for survival and adaptive behavior in a challenging world. Adapt. Behav. 16, 385-399.

Kuffler, S. W., and Nicholls, J. G. (1966). The physiology of Neuroglia cells. Ergebn. Physiol. Biol. Chem. Exp. Pharmacol. 57, 1-90.

Kuga, N., Sasaki, T., Takahara, Y., Matsuki, N., and Ikegaya, Y. (2011). Large-scale calcium waves traveling through astrocytic networks in vivo. J. Neurosci. 31, 2607-2614

Kwok, J. C. F., Dick, G., Wang, D., and Fawcett, J. W. (2011). Extracellular matrix and perineuronal nets in CNS repair. Dev. Neurobiol. 71, 1073-1089.

Lewis, T. J., and Rinzel, J. (2000). Self-organized synchronous oscillations in a network of excitable cells coupled by gap junctions. Comput. Neural Sys. 11, 299-320.

Mandelbrot, B. (1999). Multifractals and $1 / f$ Noise. New York, NY: Springer.

Marder, E., and Calabrese, R. L. (1996). Principles of rhythmic motor pattern generation. Physiol. Rev. 76, 687-717.

Marder, E., and Thirumalai, V. (2002). Cellular, synaptic and network effects of neuromodulation. Neural Netw. 15, 479-493. 
Marom, S. (2010). Neural timescales or lack thereof. Prog. Neurobiol. 90, 16-28.

Mitterauer, B. J. (2010). Significance of the astrocyte domain organization for qualitative information structuring in the brain. Adv. Biosci. Biotech. 1, 391-397.

Mitterauer, B. J. (2012). Qualitative information processing in tripartite synapses: a hypothetical model. Cogn. Comput. 4, 181-194.

Mitterauer, B. J., and KoflerWestergren, B. (2011). Possible effects of synaptic imbalances on oligodendrocyte-axonic interactions in schizophrenia: a hypothetical model. Front. Psychiatry 2:15. doi: 10.3389/fpsyt. 2011.00015

Montroll, E., and Shlesinger, M. (1982). On $1 / \mathrm{f}$ noise and other distributions with long tails. Proc. Natl. Acad. Sci. U.S.A. 79, 3380-3383.

Nadkarni, S., Jung, P., and Levine, H. (2008). Astrocytes optimize the synaptic transmission of information. PLoS Comput. Biol. 4:e1000088. doi: 10.1371/journal. pcbi. 1000088

Nassel, D. R. (2009). Neuropeptide signaling near and far: how localized and timed is the action of neuropeptides in brain circuits? Invert. Neurosci. 9, 57-75.

Nicholson, C. (1980). The dynamics of brain-cell microenvironment. Neurosci. Res. Program Bull. 18, 175-222.

Nusbaum, M. P. (2002). Regulating peptidergic modulation of rhythmically active neural circuits. Brain Behav. Evol. 60, 378-387.

Oberheim, N. A., Tian, G.-F., Han, X., Peng, W., Takano, T., Ransom, B., et al. (2008). Loss of astrocytic domain organization in the epileptic brain. J. Neurosci. 28, 3264-3276.

Ogata, K., and Kosaka, T. (2002). Structural and quantitative analysis of astrocytes in the mouse hippocampus. Neuroscience 113, 221-233.

Parpura, V., and Haydon, P. G. (2000). Physiological astrocytic calcium levels stimulate glutamate release to modulate adjacent neurons. Proc. Natl. Acad. Sci. U.S.A. 97, 8629-8634.

Pawlak, V., Wickens, J. R., Kirkwood, A., and Kerr, J. N. D. (2010). Timing is not everything: neuromodulation opens the STDP gate. Front. Synaptic Neurosci. 2:146. doi: 10. 3389/fnsyn.2010.00146

Perea, G., and Araque, A. (2010). Glia modulates synaptic transmission. Brain Res. Rev. 63, 93-102.

Perea, G., Navarrete, M., and Araque, A. (2009). Tripartite synapses: astrocytes process and control synaptic information. Trends Neurosci. 32, 421-431.

Pereira, A. Jr., and Furlan, F. A. (2010). Astrocytes and human cognition: modeling information integration and modulation of neuronal activity. Prog. Neurobiol. 92, 405-420.

Proekt, A., Brezina, V., and Weiss, K. R. (2004). Dynamical basis of intentions and expectations in a simple neuronal network. Proc. Natl. Acad. Sci. U.S.A. 101, 9447-9452.

Ribrault, C., Sekimoto, K., and Triller, A. (2011). From the stochasticity of molecular processes to the variability of synaptic transmission. Nat. Rev. Neurosci. 12, 375-387.

Rusakov, D. A., and Kullmann, D. M. (1998). Geometric and viscous components of the tortuosity of the extracellular space in the brain. Proc. Natl. Acad. Sci. U.S.A. 95, 8975-8980.

Simon, A., Olah, S., Molnar, G., Szabadics, J., and Tamas, G. (2005). Gap junctional coupling between neuroglia form cells and various interneuron types in the Neocortex. J. Neurosci. 25, 6278-6285.

Smith, M., and Pereda, A. E. (2003) Chemical synaptic activity modulates nearby electrical synapses. Proc. Natl. Acad. Sci. U.S.A. 100, 4849-4854.

Stanley, H. E., and Meakin, P. (1988). Multifractal phenomena in physics and chemistry. Nature 335, 405-409.

Stern, E., Fort, T. J., Miller, M. W., Peskin, C. S., and Brezina, V. (2007). Decoding modulation of the neuromuscular transform. Neurocomputing 70, 1753-1758.

Theodosis, D. T., Poulain, D. A., and Oliet, S. H. R. (2008). Activitydependent structural and functional plasticity of astrocyte-neuron interactions. Physiol. Rev. 88, 983-1008.

Traub, R. D., Schmitz, D., Jefferys, G. R., and Draguhn, A. (1999). High-frequency population oscillations are predicted to occur in hippocampal pyramidal neuronal networks interconnected by axoaxonal gap junctions. Neuroscience 92 , 407-426.

Ullah, G., Cressman, J. R., Barreto, E., and Schiff, S. J. (2009). The influence of sodium and potassium dynamics on excitability, seizures, and the stability of persistent states: II. Network and glial dynamics. Comput. Neurosci. 26, 171-183.

Verkhratsky, S., and Butt, A. (2007). Glia Neurobiology. New York, NY: Wiley.

Vizi, E. S., and Labos, E. (1991). Non-synaptic interactions at presynaptic level. Prog. Neurobiol. 37, 145-163.

Volterra, A., and Meldolesi, J. (2005). Astrocytes, from brain glue to communication elements: the revolution continues. Nat. Rev. Neurosci. 6, 626-640.

Werner, G. (2005). Neural Computation in Excitable Media. Available online at: http://cogprints.org/8022/7/ ExcitableMediaSeminar.pdf

Werner, G. (2010). Fractals in the nervous system: conceptual implications for theoretical neuroscience. Front. Physiol. 1:15. doi: 10.3389/ fphys.2010.00015

Werner, G. (2011). Letting the brain speak for itself. Front. Physiol. 2:60. doi: 10.3389/fphys.2011.00060

West, B. J. (2010). Fractal physiology and the fractional calculus: a perspective. Front. Physiol. 1:12. doi: 10. 3389/fphys.2010.00012

West, B. J., and Grigolini, P. (2010). Chipping away at memory. Biol. Cybern. 103, 167-174.

Zoli, M., and Agnati, L. F. (1996). Wiring and volume transmission in the central nervous system: the concept of closed and open synapses. Prog. Neurobiol. 49, 363-380.

Conflict of Interest Statement: The authors declare that the research was conducted in the absence of any commercial or financial relationships that could be construed as a potential conflict of interest.

Received: 22 January 2013; accepted: 22 February 2013; published online: 26 March 2013

Citation: Werner G and Mitterauer BJ (2013) Neuromodulatory systems. Front. Neural Circuits 7:36. doi: 10.3389/fncir. 2013.00036

Copyright (c) 2013 Werner and Mitterauer. This is an open-access article distributed under the terms of the Creative Commons Attribution License, which permits use, distribution and reproduction in other forums, provided the original authors and source are credited and subject to any copyright notices concerning any third-party graphics etc. 